\title{
Anomalous Exciton Spectra of Laser-Driven Semiconductor Superlattices
}

\author{
Kenta Yashima ${ }^{a}$, Kotaro Oka ${ }^{a}$, Ken-ichi Hino ${ }^{\text {b,c,*, }}$, \\ Nobuya Maeshima $^{\mathrm{b}, \mathrm{c}}$, and Xiao Min Tong ${ }^{\mathrm{c}, \mathrm{d}}$ \\ a Center for Life Science and Technology, School of Fundamental Science and \\ Technology, Keio University, Yokohama, Kanagawa 223-8522, Japan \\ ${ }^{\mathrm{b}}$ Doctoral Program in Frontier Science, Graduate School of Pure and Applied \\ Sciences, University of Tsukuba, Tsukuba, Ibaraki 305-8573, Japan \\ ${ }^{\mathrm{c}}$ Center for Computational Sciences, University of Tsukuba, Tsukuba, Ibaraki \\ 305-8577, Japan \\ ${ }^{\mathrm{d}}$ Doctoral Program in Materials Science, Graduate School of Pure and Applied \\ Sciences, University of Tsukuba, Tsukuba, Ibaraki 305-8573, Japan
}

\begin{abstract}
The quasienergy structure of excitonic Floquet states of laser-driven semiconductor superlattices is examined. To understand the detail of it, linear absorption spectra of optical interband transitions invoked by an alternative monochromatic probe laser are calculated based on the Liouvílle equation. It is found that for a strong driving laser, the interminiband interaction, namely, the ac-Zener tunneling, causes the red shift of the spectral peak pertaining to the $1 s$-exciton Floquet state, and enlarges the intensity of its concomitant replica bands. In particular, it is noted that one of the replicas exhibits the anomalous negative absorption.
\end{abstract}

Key words: A. Semiconductors, A. Quantum Wells, D. Optical properties $P A C S:$ 78.67.Pt

\section{Introduction}

It is known that the Floquet system, namely, the quantum system involving periodic time-dependence due to interactions with external fields such as acelectric and ac-magnetic fields exhibits the characteristic phenomenon, termed

* Corresponding author. Tel/fax: +81-29-853-4994

Email address: hino@bk.tsukuba.ac.jp (Ken-ichi Hino). 
dynamic localization (DL), where an appropriately designed periodic drive brings the quantum transport and diffusion to an almost complete standstill [1]. The DL has been the research subject of diverse interdisciplinary fields pertinent to, for instance, quantum driven tunneling of semiconductor heterostructures [2,3], optical superlattices (SLs) [4] and molecular vibrational states [1], coherent control of atomic hyperfine and Zeeman level structures $[5,6]$, and quantum chaos of a kicked rotator $[7,8]$. In this Letter, we focus exclusively on the laser-driven semiconductor SLs, termed dynamic WannierStark ladder (DWSL) $[2,3]$. It was demonstrated that the wave packet dynamics of the DWSL shows spatial charge localization corresponding to the DL, which is featured by the collapse of the quasienergy miniband. Physics underlying the DWSL is enriched by additional complexity of an exciton (EX) effect [9], electron correlation [10], random disorder [11], nonlinear coherent dynamics [12], and interminiband interactions due to the Zener tunneling (ZT) [13].

In the preceding paper [14], the linear photoabsorption spectra of the the non-EX-DWSL were calculated to find the pronounced spectral modulation, in particular, the unexpected dent structure arising from the ac-ZT between photon sidebands pertaining to different minibands, when the driving laser $F(t)$ - with time $t$ - was relatively strong. This was much different from the corresponding ac-ZT-free case. Here, the alternative monochromatic laser $f_{p}(t)$ as a probe was exerted on the laser-driven SLs, causing the optical interband transitions. To deepen the understanding of the DWSL Floquet state at the more realistic level, the present paper is aimed at the exploration of interplay between the EX effect and the ac-ZT. For this purpose, the linear absorption spectra of the DWSL in view of the EX effect under relatively strong $F(t)$ are evaluated. As will be shown later in detail, the anomalous negative absorption appears, which results from the synergy between the EX effect of concern here and the ac-ZT. Such an anomaly is not found without either of these two effects. Actually, in Ref. [14], the negative absorption was not observed in the spectra because of no EX effect included: the significance of this effect was just suggested for the appearance of the anomaly.

\section{Formulation}

The total Hamiltonian of the system concerned comprises the joint-miniband SL Hamiltonian composed of the conduction $(c)$-band and valence $(v)$-band field-free Hamiltonians, the Coulomb interaction between electrons, the intersubband interaction caused by the driving laser $F(t)$, and the interband interaction invoked by the probe laser $f_{p}(t)$, where these two electric fields at time $t$ are represented by $F(t)=F_{a c} \cos (\omega t)$ (with $F_{a c}$ as amplitude and $\omega$ as frequency), and $f_{p}(t)=f_{p 0} \cos \left(\omega_{p} t\right)$ (with $f_{p 0}$ as amplitude and 
$\omega_{p}$ as frequency). The formulation developed here is directed toward seeking the microscopic polarization of the interband transition, $p_{\lambda \lambda^{\prime} \mathbf{K}_{\|}}(t)$, defined by $p_{\lambda \lambda^{\prime} \mathbf{K}_{\|}}(t) \equiv\left\langle a_{\lambda \mathbf{K}_{\|}}^{(v) \dagger} a_{\lambda^{\prime} \mathbf{K}_{\|}}^{(c)}\right\rangle$, by solving the Liouvílle equation [15] of the present system, based on the same theoretical framework as made in Ref. [14]. Here, $\lambda^{(\prime)}$ represents the lump of the SL miniband index $b^{(\prime)}$ and the SL lattice site $l^{(\prime)}$, namely, $\lambda^{(\prime)}=\left(b^{(\prime)}, l^{(\prime)}\right)$, and $\mathbf{K}_{\|}$is the in-plane momentum of a pair of the electrons of the $c$ and $v$ bands, where this is associated with the relative motion of these two electrons in the plane normal to the direction of crystal growth (the $z$-axis). Furthermore, $a_{\lambda \mathbf{K}_{\|}}^{(s) \dagger}\left(a_{\lambda \mathbf{K}_{\|}}^{(s)}\right)$ represents the creation (annihilation) operator of the electron with $\lambda$ and $\mathbf{K}_{\|}$in the band $s$, satisfying the usual anti-commutation relation, and $\langle\cdots\rangle$ has been meant by taking an expectation value.

The main approximations made here are recapitulated in the following. (i) The nearest-neighbor tight-binding (NNTB) model for the the $c$ - and $v$-band SL Hamiltonians is employed. (ii) The Wannier function, $\langle z \mid \lambda\rangle$, at the position $z-l d$ in miniband $b$ with $d$ as the lattice constant of the concerned SLs, is approximated by a corresponding wave function of a single quantum-well with an infinite potential barrier. (iii) The Coulomb interaction for an EX composed of only a single electron-hole pair is retained, whereas the many-body Coulomb correlation effect is neglected. (iv) It is assumed that the probe laser is weak enough to satisfy the relation $F_{a c}>>f_{p 0}$ and that $\omega_{p}$ is much greater than $\omega$, namely, $\omega<<\omega_{p}$. Thus, $F(t)$ does not contribute to the interband transitions and $f_{p}(t)$ does not contribute to the intersubband transitions. The resulting the absorption coefficient is linear in $f_{p}(t)$, however non-linear in $F(t)$. The validity and criteria of the applicability of these approximations were already discussed in Ref. [14]. Hereafter, the atomic units are used throughout unless otherwise stated.

The exact expression of the absorption coefficient $\alpha_{a b s}^{(e x)}\left(\omega_{p} ; \omega\right)$ for the interband optical transition between "time-dependent" Floquet states is not trivial a priori, differing from that between usual time-independent steady states. For the purpose of deriving it in an ab initio way, we begin with the Liouvílle equation under the approximation (iii) in addition to the other approximations mentioned above. The Liouvílle equation to be solved is reduced to

$$
\begin{aligned}
& i\left(\frac{d}{d t}+\gamma-i \omega_{p}\right) \bar{p}\left(\boldsymbol{\rho}, z_{v}, z_{c}, t\right)+(2 \pi)^{2} e^{i \omega_{p} t} f_{p}^{(+)}(t) d_{0}^{(v c)} \delta(\boldsymbol{\rho}) \delta\left(z_{v}-z_{c}\right) \\
= & \int d z\left[\bar{p}\left(\boldsymbol{\rho}, z_{v}, z, t\right) H^{(c)}\left(z, z_{c}, t\right)-H^{(v)}\left(z_{v}, z, t\right) \bar{p}\left(\boldsymbol{\rho}, z, z_{c}, t\right)\right] \\
& +\mathcal{H}\left(\boldsymbol{\rho}, z_{v}, z_{c}\right) \bar{p}\left(\boldsymbol{\rho}, z_{v}, z_{c}, t\right),
\end{aligned}
$$

where in place of $p_{\lambda \lambda^{\prime} \mathbf{K}_{\|}}(t)$, the positional representation of the microscopic 
polarization, $\bar{p}\left(\boldsymbol{\rho}, z_{v}, z_{c}, t\right)$, has been used, which is defined as

$$
\bar{p}\left(\boldsymbol{\rho}, z_{v}, z_{c}, t\right)=e^{i \omega_{p} t} \sum_{\lambda, \lambda^{\prime}} \int d \mathbf{K}_{\|} e^{i \mathbf{K}_{\|} \cdot \boldsymbol{\rho}}\left\langle z_{v} \mid \lambda\right\rangle p_{\lambda \lambda^{\prime} \mathbf{K}_{\|}}(t)\left\langle\lambda^{\prime} \mid z_{c}\right\rangle .
$$

In addition, the phenomenological homogeneous broadening $\gamma$ has been introduced, $d_{0}^{(v c)}$ is the interband dipole transition matrix element of a bulk material, and $f_{p}^{(+)}(t) \equiv\left(f_{p 0} / 2\right) e^{-i \omega_{p} t}$. In Eq. (1), the Hamiltonian, given by

$$
\mathcal{H}\left(\boldsymbol{\rho}, z_{v}, z_{c}\right)=-\frac{\boldsymbol{\nabla}_{\boldsymbol{\rho}}^{2}}{2 m_{\|}}-\frac{1}{\epsilon \sqrt{\boldsymbol{\rho}^{2}+\left(z_{c}-z_{v}\right)^{2}}},
$$

governs the Coulombic motion of an electron-hole pair in the $\boldsymbol{\rho}$-direction (the layer plane), with $m_{\|}$and $\epsilon$ as the in-plane reduced mass of the pair and the static dielectric constant, respectively. Further, $H^{(s)}\left(z, z^{\prime}, t\right)$ has been given by $\left\langle z\left|\hat{H}^{(s)}(t)\right| z^{\prime}\right\rangle$, with the operator $\hat{H}^{(s)}(t)$ defined as

$$
\begin{aligned}
\hat{H}^{(s)}(t)= & \sum_{\lambda=(l, b)}\left[(-1)^{b+\sigma^{(s)}} \frac{\Delta_{b}^{(s)}}{4}(|l, b\rangle\langle l+1, b|+| l+1, b\rangle\langle l, b|)+\epsilon_{0 b}^{(s)}|\lambda\rangle\langle\lambda|\right] \\
& -F(t) \frac{1}{2} \sum_{\lambda, \lambda^{\prime}}\left[|\lambda\rangle Z_{\lambda \lambda^{\prime}}^{(s)}\left\langle\lambda^{\prime}|+| \lambda^{\prime}\right\rangle Z_{\lambda^{\prime} \lambda}^{(s) *}\langle\lambda|\right] .
\end{aligned}
$$

The first term represents the Hamiltonian of the SLs for band $s$ by means of the NNTB model, where $\Delta_{b}^{(s)}$ is the width of miniband $b$ in band $s$, and $\epsilon_{0 b}^{(c / v)}$ is the center of miniband $b$ reckoned from the bottom/top of the $c / v$ band, with $\sigma^{(c)}=0$ and $\sigma^{(v)}=1$. Further, the second term stands for the dipole interaction with $F(t)$, and the dipole matrix element is given by $Z_{\lambda \lambda^{\prime}}^{(s)}=$ $l d \delta_{\lambda \lambda^{\prime}}+X_{b b^{\prime}}^{(s)} \delta_{l l^{\prime}}\left(1-\delta_{b b^{\prime}}\right)$, where it is noted that $X_{b b^{\prime}}^{(s)}$ causes the ac-ZT to be stressed herein.

Equation (1) is the inhomogeneous equation corresponding to the homogeneous equation for the joint-miniband EX-DWSL Floquet wavefunctions $\psi_{\nu}\left(\boldsymbol{\rho}, z_{v}, z_{c}, t\right)$, given by

$$
\begin{aligned}
& \left(i \frac{d}{d t}+E_{\nu}\right) \psi_{\nu}\left(\boldsymbol{\rho}, z_{v}, z_{c}, t\right) \\
= & \int d z\left[\psi_{\nu}\left(\boldsymbol{\rho}, z_{v}, z, t\right) H^{(c)}\left(z, z_{c}, t\right)-H^{(v)}\left(z_{v}, z, t\right) \psi_{\nu}\left(\boldsymbol{\rho}, z, z_{c}, t\right)\right] \\
& +\mathcal{H}\left(\boldsymbol{\rho}, z_{v}, z_{c}\right) \psi_{\nu}\left(\boldsymbol{\rho}, z_{v}, z_{c}, t\right),
\end{aligned}
$$

where $E_{\nu}$ is the quasienergy and $\psi_{\nu}\left(\boldsymbol{\rho}, z_{v}, z_{c}, t+T\right)=\psi_{\nu}\left(\boldsymbol{\rho}, z_{v}, z_{c}, t\right)$ with $T=2 \pi / \omega$. Therefore, it is plausible to expand $\bar{p}\left(\boldsymbol{\rho}, z_{v}, z_{c}, t\right)$ as $\bar{p}\left(\boldsymbol{\rho}, z_{v}, z_{c}, t\right)=$ 
$\sum_{\nu} \psi_{\nu}\left(\boldsymbol{\rho}, z_{v}, z_{c}, t\right) a_{\nu}$ in terms of the basis set $\left\{\psi_{\nu}\left(\boldsymbol{\rho}, z_{v}, z_{c}, t\right)\right\}$. Using the orthonormality relation of this set yields the explicit form of the expansion coefficient $a_{\nu}$ as follows:

$$
a_{\nu}=\left[E_{\nu}-\omega_{p}-i \gamma\right]^{-1} \frac{(2 \pi)^{2} d_{0}^{(v c) *}}{T} \int_{0}^{T} d t e^{i \omega_{p} t} f_{p}^{(+)}(t) \bar{\psi}_{\nu}(t)^{*}
$$

where $\bar{\psi}_{\nu}(t) \equiv \int d z\left[\psi_{\nu}(\mathbf{0}, z, z, t)\right]$. The price to be paid for such a simple form of $a_{\nu}$ leads to requirement of a rather involved evaluation of Eq. (5). To this end, $\psi_{\nu}$ is represented by

$$
\psi_{\nu}\left(\boldsymbol{\rho}, z_{v}, z_{c}, t\right)=\sum_{k, B, J, i} \Phi_{[B J]}^{*}\left(k ; z_{v}, z_{c}, t\right) \varphi_{i}(\boldsymbol{\rho}) C_{k B J i, \nu} .
$$

$\Phi_{[B J]}$ is the ZT-free and non-EX joint-miniband DWSL Floquet wavefunction defined as $\Phi_{[B J]}^{*}\left(k ; z_{v}, z_{c}, t\right)=\phi_{b_{v} j_{v}}^{(v)}\left(k ; z_{v}, t\right) \phi_{b_{c} j_{c}}^{(c) *}\left(k ; z_{c}, t\right)$, where $B$ is the index of the joint-miniband, namely, $B=\left(b_{c}, b_{v}\right)$, $J$ is the index of the photon sideband, namely, $J \equiv j_{c}-j_{v}$, and $\phi_{b j}^{(s)}(k ; z, t)$ is the Houston wavefunction [16] for photon sideband $j$ and miniband $b$ of band $s$ at the joint Bloch momentum $k$. Moreover, $\varphi_{i}(\boldsymbol{\rho})$ stands for the piecewise basis function at grid $i$ in the $\boldsymbol{\rho}$ direction by virtue of the discrete variable representation (DVR) [17]. Equation (5) can be solved by resorting to the standard diagonalization procedure, and hence a set of the coefficients $\left\{C_{k B J i, \nu}\right\}$ is obtained.

Once Eq. (1) is solved, the linear optical susceptibility $\chi(t)$ with respect to $f_{p}^{(+)}(t)$ is provided by

$$
\chi(t)=\frac{1}{\epsilon_{0}}\left|d_{0}^{(v c)}\right|^{2} \sum_{\nu} \frac{\mathcal{O}_{\nu}(t)}{E_{\nu}-\omega_{p}-i \gamma},
$$

where $\mathcal{O}_{\nu}(t) \equiv \bar{\psi}_{\nu}(t) \frac{1}{T} \int_{0}^{T} d t^{\prime} \bar{\psi}_{\nu}^{*}\left(t^{\prime}\right)$, and $\epsilon_{0}$ is the dielectricity of vacuum. Accordingly, the absorption coefficient becomes of the form [14]:

$$
\alpha_{a b s}^{(e x)}\left(\omega_{p} ; \omega\right)=\frac{\omega_{p}}{c} \sum_{J} \operatorname{Im} \chi_{J}\left(\omega_{p} ; \omega\right),
$$

where $\chi(t) \equiv \sum_{J} e^{i J \omega t} \chi_{J}\left(\omega_{p} ; \omega\right)$, and $c$ is the speed of light. Note that $\operatorname{Im} \chi_{0}\left(\omega_{p} ; \omega\right) \geq$ 0 .

\section{Results and Discussion}

The sample of the SLs for the present calculations is GaAs $/ \mathrm{Ga}_{0.75} \mathrm{Al}_{0.25} \mathrm{As}$ of $35 / 11$ monolayers (ML) $[1 \mathrm{ML}=2.83 \AA]$ for the well and barrier thickness, consisting of ten quantum wells. The material parameters employed here are 


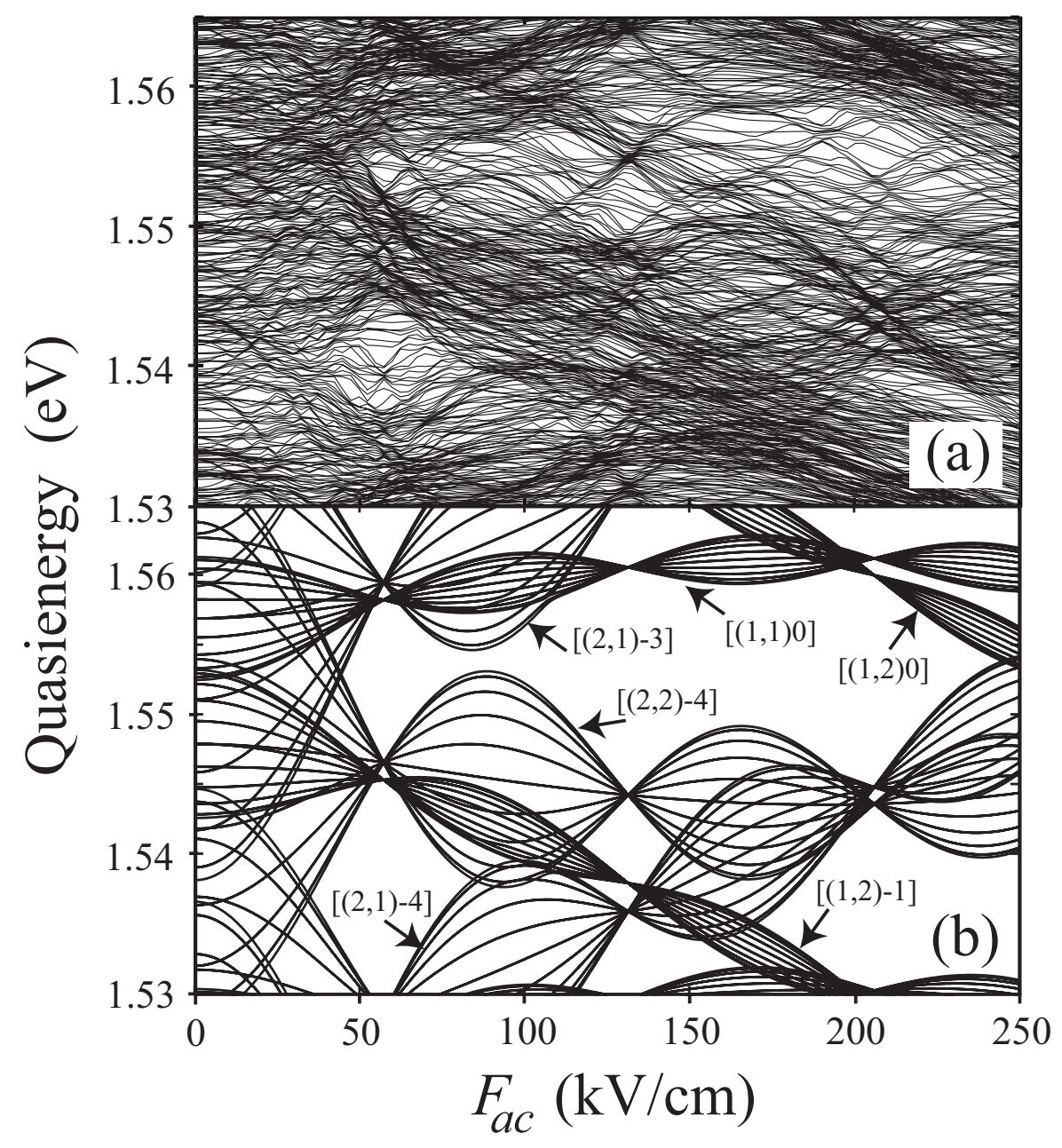

Fig. 1. The quasienergy $E_{\nu}(\mathrm{eV})$ of the DWSL Floquet state as a function of the peak electric field $F_{a c}(\mathrm{kV} / \mathrm{cm})$ of the driving laser. The panels (a) and (b) show the results with and without the EX effect, respectively. In panel (b), the main component of the Houston state is denoted as $\left[\left(b_{c}, b_{v}\right) J\right]$ in every tilted gourd-shaped quasienergy band.

given in Ref. [14]. The calculations have been implemented within the four joint-miniband model, by incorporating ten photon sidebands and ten DVRfunctions as the expansion basis set for $\psi_{\nu}$. Furthermore, $\omega$ is set equal to $\left[\epsilon_{02}^{(c)}-\epsilon_{01}^{(c)}\right] / 3$, namely, $\omega=31 \mathrm{meV}$ so that the band $\left[\left(1, b_{v}\right) J\right]$ is resonant with the band $\left[\left(2, b_{v}\right) J\right]$ by three photon absorption.

The quasienergies, $E_{\nu}$, obtained by solving Eq. (5) are shown in Fig. 1 (a) as a function of $F_{a c}$, while Fig. 1 (b) is the results for the non-EX-DWSL with the ac-ZT for comparison with the panel (a). In Fig. 1 (b), the main component of the Houston state $[B J]$ is designated as $\left[\left(b_{c}, b_{v}\right) J\right]$ in the respective tilted gourd-shaped quasienergy bands. The anticrossing arises from the acZT between states with different $B \mathrm{~s}$ and $J_{\mathrm{S}}$, however, with the same $k$ : for instance, the bands of $[(1,1) 0]$ and $[(1,2)-1]$ are repelled strongly. On the 


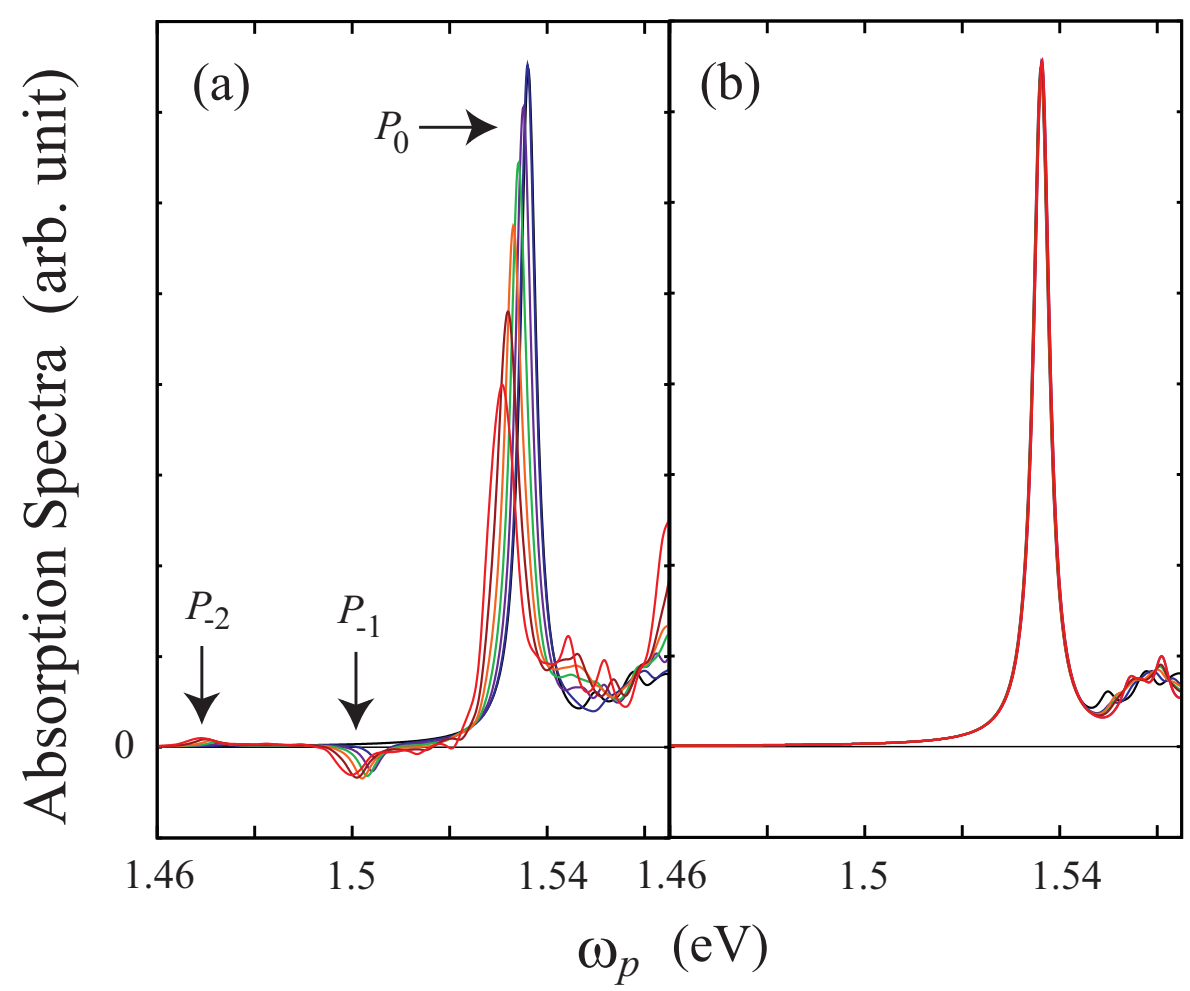

Fig. 2. The absorption spectra $\alpha_{a b s}^{(e x)}\left(\omega_{p} ; \omega\right)$ with $\gamma=2(\mathrm{meV})$ as a function of $\omega_{p}(\mathrm{eV})$ at several values of $F_{a c}(\mathrm{kV} / \mathrm{cm}): F_{a c}=0$ (black), 28.75 (blue), 57.5 (purple), 75 (green), 92.5 (orange), 112.5 (brown), 132.5 (red). The panels (a) and (b) show the results with and without the ac-ZT, respectively. In panel (a), the main peak of the $1 s$-EX Floquet state, the dip of the first replica, and the small peak of the second replica are denoted as $P_{0}, P_{-1}$, and $P_{-2}$, respectively.

other hand, the structure of $E_{\nu}$ of the panel (a) looks more intricate than that of the panel (b) due to the EX effect causing entangled anticrossings among all Houston states. The sequence of the EX bound states is located right below the lower-edges of every tilted gourd-shaped quasienergy band seen in Fig. 1 (b), whereas the EX (pseudo)continuum states are allowed to spread over all energies above these band edges: thus the DL seen clearly in the panel (b) is not discernible in the panel (a) any longer.

Figure 2 shows the EX spectra, $\alpha_{a b s}^{(e x)}\left(\omega_{p} ; \omega\right)$, as a function of $\omega_{p}$ at several $F_{a c}$ 's ranging from 0 to $130 \mathrm{kV} / \mathrm{cm}$, where the spectra of the panels (a) and (b) are the results with and without the ac-ZT, respectively. The numerical accuracy has been checked by changing the number of the bases for the photon sideband and the DVR. It is ensured that the calculated spectra almost converge for $\omega_{p}<1.56 \mathrm{eV}$, though they still depend on the number of the bases included here for $\omega_{p}>1.56 \mathrm{eV}$. The numerical convergence would entail the heavier burden with $F_{a c}$ greater, especially, for $F_{a c}>150 \mathrm{kV} / \mathrm{cm}$. The salient peaks seen around $\omega_{p}=1.53 \mathrm{eV}$ are assigned to the $1 \mathrm{~s}$ EX Floquet state pertaining to the $[(1,1) 0]$ band marked in Fig. 1 (b). It is seen that the position of this 
peak [denoted as $P_{0}$, in Fig. 2 (a)] shows the red shift and the peak intensity decreases, as $F_{a c}$ becomes large. Note that the above-mentioned red shift does not result from the well-known bandgap renormalization observed in the nonlinear optical interband transition for $f_{p 0}>>1$, which is much contrasted with the present case of $f_{p 0}<<1$. Moreover, the additional dip [denoted as $P_{-1}$ ] and peak [denoted as $P_{-2}$ ] become pronounced around $\omega_{p}=1.5$ and 1.47 $\mathrm{eV}$, respectively, and move toward the lower-energy side coincidently with the red shift of $P_{0}$. Since the energy difference of $P_{0}$ from $P_{-n}$ is approximately identical to $n \omega$ with $n=1,2$, it is understood that both $P_{-1}$ and $P_{-2}$ are manifested as the replica bands of the parent band $P_{0}$, resulting from the relatively strong ac-ZT presumably between the bands of $[(1,1) 0]$ and $[(1,2)-$ 1]. This tendency is much contrasted with that seen in Fig. 2 (b), where the position of the main peak remains almost unaltered without any additional structure.

Furthermore, it is worthwhile to note that $P_{-1}$ exhibits the negative absorption, namely, the optical gain. Obviously, this anomaly is caused by the interplay of the EX effect and the ac-ZT, and both of the effects are indispensable for its manifestation. Actually, the spectra without the EX effect, which was reported in the preceding paper [14], show no such a signal. Further analysis indicates that the anomaly concerned is attributed to the non-linear optical interminiband transitions accompanying the non-zero net energy exchange, $J \omega(J \neq 0)$, due to the ac-ZT. The negative absorption was also discussed in other studies $[18,19]$, where the SLs subjected to relatively weak static- and alternating-electric fields were probed by the short-pulse optical response, differing from the present case of the weak monochromatic-probe response of $f_{p}(t)$. The origin of this gain is considered the EX effect and the many-body Coulomb effect of carriers due to the short-pulse excitation. In this sense, the negative absorption found in the present paper has the different origin, and thus this would be regarded as a novel effect.

\section{Conclusion}

The quasienergy structure of the EX Floquet states in the laser-driven SLs are investigated by examining the interband linear absorption spectra, and the red shift of the $1 s$-EX peak and the formation of the concomitant replica bands are confirmed. In particular, one of these replicas shows the anomalous negative absorption due to the ac-ZT and the EX effect. It is remarked that the negative absorption is realized without population inversion of the SL system, similarly to the well-known lasing without population inversion in the driven atomic Lambda system [20], though the origin is different from the present one. In addition, it is commented that the recent experiment observed unusual behavior of the interband absorption spectra in bulk semiconductors strongly 
driven by intense ultrashort midinfrared laser fields [21]. Further, impurity states in the low-dimensional system such as semiconductor quantum wells and dots under intense $\mathrm{THz}$ laser fields were studied using the dressed-band approach [22].

This research was financially supported by Grant-in-Aid for Scientific Research (C) [No.19540413] from the Japan Society for the Promotion of Science.

\section{References}

[1] M. Grifoni and P. Hänggi, Phys. Rep. 304 (1998) 229.

[2] M. Holthaus, Phys. Rev. Lett. 69 (1992) 351.

[3] J. Zak, Phys. Rev. Lett. 71 (1993) 2628.

[4] K. W. Madison, M. C. Fischer and M. G. Raizen, Phys. Rev. A 60 (1999) R1767.

[5] S. Haroche, C. Cohen-Tannoudji, C. Audoin, and J. P. Schermann, Phys. Rev. Lett. 24 (1970) 861.

[6] G. Xu and D. J. Heinzen, Phys. Rev. A 59 (1999) R922.

[7] H-J. Stöckman, Quantum Chaos: An Introduction (Cambridge, UK, 1999) Chap. 4, p. 135.

[8] M. Glück, A. R. Kolovsky and H. J. Korsch, Phys. Rep. 366 (2002) 103.

[9] K. Yashima, K. Hino, and N. Toshima, Phys. Rev. B 68 (2003) 235325.

[10] Z. -G. Wang, D. Suqing, and X. -G. Zhao, Phys. Lett. A 353 (2006) 210.

[11] M. Holthaus, G. H. Ristow, and D. W. Hone, Phys. Rev. Lett. 75 (1995) 3914.

[12] K. A. Pronin, P. Reinecker, and A. D. Bandrauk, Phys. Rev. B 71 (2005) 195311.

[13] K. Hino, K. Yashima, and N. Toshima, Phys. Rev. B 71 (2005) 115325.

[14] K. Hino, X. M. Tong, and N. Toshima, Phys. Rev. B 77 (2008) 045322.

[15] H. Haug and S. W. Koch, Quantum Theory of the Optical and Electronic Properties of Semiconductors, Third Edition, (World Scientific, Singapore, 1994) Chap. 12, p. 218.

[16] W. V. Houston, Phys. Rev. 57 (1940) 184.

[17] K. Hino, J. Phys. Soc. Jpn. 67 (1998) 3159.

[18] T. Meier, H. J. Kolbe, A. Thränhardt, G. Weiser, and S. W. Koch, Physica E, 7, (2000) 267. 
[19] M. M. Dignam, Phys. Rev. B 59 (1999) 5770.

[20] Z. Ficek and S. Swain, Quantum Interference and Coherence: Theory and Experiments (Springer, New York, 2005) Chap. 5, p. 201.

[21] A. Srivastava, R. Srivastava, J. Wang, and J. Kono, Phys. Rev. Lett. 93 (2004) 157401.

[22] L. E. Oliveira, A. Latge, and H. S. Brandi, Phys. Status Solidi a 190 (2002) 667. 